\title{
Desarrollo y perspectivas de la medicina tradicional china: reflexiones a nivel local y global ${ }^{1}$
}

\section{Development and Perspectives of Traditional Chinese Medicine: Local and Global Reflections}

\author{
MARÍA FLORENCIA INCAURGARAT \\ Universidad Nacional de Mar del Plata, \\ Argentina
}

Resumen: El presente artículo explora tres ejes en relación con la medicina tradicional china a nivel local y global. En un primer momento, se realiza una genealogía de este saber milenario, desde sus orígenes (registrados) hasta la actualidad, con énfasis en los encuentros con la llamada "medicina occidental" y en cómo su llegada terminó por fortalecer el saber nativo y crear una "nueva geografía”. En segundo lugar, se exploran las diversas formas como los ciudadanos chinos se apropian de ambos sistemas médicos en la China contemporánea. Por último, se hace un acercamiento a la expansión global de este saber, por medio de su comoditización o como recurso potencial de

Recepción: 26 de junio de 2020. / Aceptación: 11 de febrero de 2021.

${ }^{1}$ Para la realización de este trabajo se contó con una beca del Programa de Jóvenes Sinólogos Visitantes otorgada por el gobierno chino en 2018. 
soft power. De esta forma, se pretende colaborar en un campo poco explorado desde las ciencias sociales, sobre este corpus de prácticas y conocimientos, su dinamismo y su constante modificación.

Palabras clave: medicina tradicional china; medicina occidental; encuentros; China contemporánea; perspectiva local-global.

Abstract: This article explores three components of traditional Chinese medicine, both locally and globally. First, the text provides a genealogy of this ancient knowledge, starting from its (recorded) origins and continuing to the present day, emphasizing the encounters with so-called Western medicine, highlighting how the arrival of the latter ended up strengthening the native knowledge and creating a "new geography." Second, it explores the various ways in which Chinese citizens appropriate both medical systems in contemporary China. The article concludes with an analysis of the global expansion of this knowledge, through its commoditization or as a potential resource of soft power, thus contributing to a field rarely explored by the social sciences by examining these practices and areas of knowledge, their dynamism and constant modification.

Keywords: Chinese traditional medicine; Western medicine; encounters; contemporary China; local-global perspective.

\section{Introducción}

En el contexto de los profundos cambios que han tenido lugar en China durante las últimas décadas, y especialmente durante los últimos años, no sólo en relación con la economía, sino también en lo relativo a aspectos socioculturales, es necesario considerar aquí uno de esos aspectos: su sistema médico tradicional. Muchos debates e investigaciones relacionados con el desarrollo de China han surgido desde diferentes áreas de estudio, principalmente de campos como la economía, la política y los estudios internacionales. Sin embargo, es escasa la literatura en el ámbito de las ciencias sociales, sobre todo en español, respecto del desarrollo de la medicina tradicional china 
en este contexto cambiante. De esta forma, los modos en que la población china se apropia de esta práctica milenaria, y cómo ésta se orienta y se desarrolla en la "nueva era", ${ }^{2}$ se convierten en una amplia área por explorar.

En un primer punto de análisis, se explorará la herencia y el desarrollo de este sistema médico, incluidas las formas en que coexiste en la China contemporánea con el llamado sistema médico "occidental", el alcance de la influencia mutua y las formas en que los ciudadanos chinos se apropian de ambos. Para este propósito, en primer lugar, se rastrearán los caminos de la medicina tradicional china a partir de sus orígenes (registrados), y luego, los encuentros que se fueron sucediendo con la "medicina occidental"' hasta la actualidad. Todo esto se sostendrá mediante la investigación bibliográfica (libros, artículos académicos, comunicados de prensa, etc.) y un breve trabajo de campo realizado en Hangzhou, ${ }^{4}$ China, durante septiembre de 2018. Esta estancia brindó la posibilidad de experimentar de primera mano la dinámica y la coexistencia

${ }^{2}$ Este concepto surgió durante el XIX Congreso del Partido Comunista Chino, celebrado en octubre de 2017, en el cual se estableció la teoría política denominada "Pensamiento de Xi Jinping sobre el socialismo con características chinas para una nueva era”. Aquí, tomado como término nativo, representa un nuevo capítulo para la historia china, después de Mao Zedong y Deng Xiaoping, encarnado en la figura de Xi Jinping. Este concepto está asociado a los 14 principios y valores políticos anunciados por Xi durante el congreso, en relación con el objetivo principal del resurgimiento de la nación china como una superpotencia mundial para el 2050 (Moreno 2018).

${ }^{3}$ Aunque se puede afirmar que hay una medicina científica occidental, en el campo de la antropología de la salud se prefiere el término "biomedicina" o "modelo médico hegemónico" debido a que, en la conceptualización de "Occidente", se diluyen y homogeneizan las diversas formas de atender y concebir la salud que se dan en esta vasta región del planeta. Sin embargo, cuando aquí se utilice este término, será teniendo en cuenta la clasificación nativa entre la medicina china (zhōng ȳ 中医) y la medicina occidental ( $x \bar{\imath} y \bar{\imath}$ 西医), ampliamente difundida en la literatura especializada y los discursos nativos.

${ }^{4}$ Hangzhou es la capital de la provincia de Zhejiang, una de las más prósperas del país a pesar de ser una de las más pequeñas en cuanto a territorio. La ciudad de Hangzhou, ubicada a menos de una hora en tren bala de la megaurbe de Shanghái, cuenta con 10000000 de habitantes y está entre las 10 ciudades más grandes del país. 
de los dos sistemas de salud, el biomédico y el tradicional. En este contexto, la autora asistió a dos hospitales en dicha ciudad, así como a la Universidad de Medicina China de Zhejiang. Del mismo modo, le fue posible visitar algunas farmacias y clínicas tradicionales locales, testigos de la larga y notable tradición de la medicina tradicional en esta región.

Finalmente, considerando el desarrollo de la iniciativa One Belt One Road, ${ }^{5}$ será necesario volver a examinar algunas de estas preguntas, ya no dentro del territorio chino, sino a lo largo de este corredor con flujos globales que parten desde y hacia China, lo que también llevará a discutir sobre el encuentro de dos sistemas médicos diferentes a una escala cada vez más amplia.

\section{La medicina tradicional china y su encuentro con la medicina occidental desde una perspectiva histórica}

Aunque el primer registro escrito de una enfermedad se remonta a la dinastía Shang (1600-1100 a.e.c.), muchos historiadores ubican el origen preciso de la medicina tradicional china durante la dinastía Han (206 a.e.c.-220 e.c.), periodo en el que se hallaron registros explícitos de técnicas médicas. Se considera también que fue en ese momento en el que se redactó uno de los textos clásicos más importantes de la medicina tradicional jamás escrito: el Canon de medicina interna del Emperador Amarillo (Huángdì Nèijing 黄帝内经). Este libro ha sido el tratado más influyente durante más de 2000 años y todavía lo consultan los médicos tradicionales, ya que constituye "una fuente valiosa de inspiración teórica y conocimiento práctico en entornos clínicos modernos" (Unschuld 2003, ix). Compuesto por múltiples volúmenes, el tratado consigna las propiedades y el sabor de las medicinas junto con sus formas

5 También llamada "Nueva Ruta de la Seda”, esta iniciativa impulsada desde 2013, se trata de un megaproyecto económico que recrea y amplía a nivel global la antigua ruta de intercambio comercial. 
de prescripción, a la vez que formula nociones sobre la función del cuerpo humano y el mundo físico, las cuales siguen siendo las ideas básicas concebidas por los profesionales de la medicina tradicional. Entre ellas, por ejemplo, se encuentra la concepción de la salud como equilibrio, y la de la enfermedad como su ruptura o desbalance. A su vez, se explican conceptos fundamentales, como yinyáng 阴阳, ${ }^{6}$ los 'cinco elementos de la naturaleza' wǔxing 五行 (madera, fuego, tierra, metal, agua) y el difundido concepto de qi 气. 7 Del mismo modo, prácticas como la acupuntura o la moxibustión se describen de manera similar a las que se emplean en la actualidad. Por éstas y otras razones, se considera ampliamente que las enseñanzas de este clásico aún sostienen la práctica de la medicina tradicional (Puerta 2009).

Desde entonces, luego de miles de años de experiencia clínica, la aplicación de los métodos terapéuticos chinos ha consolidado un sistema teórico y curativo considerado como un "precioso patrimonio cultural con una larga historia y valor práctico" (Liao 2017, 17). De hecho, de las cuatro áreas de conocimiento desarrolladas en la antigua China (con sus propios sistemas de conocimiento y técnicas), es decir, la astronomía, la aritmética, la agronomía y la medicina, la última es la única que permanece sin haber sido remplazada por la ciencia occidental, y sigue desempeñando un papel importante en la vida de la mayoría de los chinos.

Aunque ambos sistemas coexisten hoy, es importante señalar que la llegada de la medicina occidental a China ha producido muchos cambios profundos en las formas de cuidar $\mathrm{y}$ atender la salud de las poblaciones. Por esa razón, es necesario rastrear sus encuentros ${ }^{8}$ en el tiempo.

${ }^{6}$ Los caracteres simplificados y la transcripción fonética en el sistema pinyin se utilizarán para nombres o conceptos en chino mandarín.

7 También conocido como chi, según el sistema Wade-Giles de romanización del chino mandarín.

${ }^{8}$ Es necesario hacer aquí una salvedad teórica y aclarar que la categoría de "encuentro" es retomada en este trabajo no desde una lente que ve el contacto entre dos o más grupos como exento de conflictos y en aparente armonía, sino más bien 
Así, se pueden hallar los primeros intercambios relacionados con la atención médica desde los días de la dinastía Han y el Imperio romano, cuando se estableció la comunicación entre China y Occidente a través de la Ruta de la Seda $(\mathrm{Fu}$ 2014). Sin embargo, el primer registro de misioneros médicos de Occidente se dio en la dinastía Tang, con los cristianos nestorianos que llegaron de Medio Oriente e introdujeron la medicina hipocrática en el 635 e.c. Aun con estos primeros encuentros, no fue hasta las guerras del Opio en el siglo XIX cuando la medicina occidental definitivamente se asentó en territorio chino, lo que acarrearía cambios profundos en la sociedad local y en las formas de prestar y recibir atención médica. A continuación se sintetizan los principales hitos que marcaron estos acercamientos.

En 1842, el Tratado de Tianjin permitía a los extranjeros viajar a cualquier lugar dentro del país sin estar sujetos a la ley china. Por primera vez, a los misioneros se les permitió poseer propiedades y residir fuera de los puertos del tratado. Aunque la prioridad de los misioneros era la evangelización —de ahí su esfuerzo por expandirse más allá de los puertos-, los hospitales y las clínicas que creaban a su paso atraían más conversos que la predicación. Así, a finales del siglo XIX, la mayoría de las ciudades tenía una misión cristiana y una "clínica de misión", donde incluyeron progresivamente programas de educación médica y educación escolar occidental. Con el tiempo, ganaron cada vez más influencia, y en 1881 se fundó la Escuela de Medicina de Tianjin como la primera que estableció el gobierno para la enseñanza de la medicina occidental (Jackson 2011).

Una vez que se fundó la República y fue abolida la última dinastía, el movimiento político liderado por el primer presidente, San Yat-Sen (médico de formación, capacitado en Occidente), intentó impulsar una modernización de la atención de la salud y recomendó la medicina occidental sobre la nativa.

como encuentros que implican una constante disputa y renegociación de identidades y posiciones de estatus en el terreno. Siguiendo a Faier y Rofel (2014), entonces, la noción de "encuentro" no remite a su sentido más convencional. 
En este contexto, un evento epidemiológico desafortunado que tuvo lugar entre 1910 y 1911, la llamada "peste manchuriana", estuvo estrictamente relacionado con la crisis del sistema médico y la consiguiente pérdida de reputación de la medicina tradicional (Lei 2000). Durante la plaga neumónica en la que murieron 60000 personas en Manchuria, casi un centenar de médicos tradicionales fueron seleccionados especialmente para controlarla, pero todos murieron a causa de ella. "La única persona que pudo ayudar fue un joven médico capacitado en Cambridge, Wu Lien-te, que utilizó su conocimiento científico en la dirección de las medidas sanitarias y de salud pública necesarias para evitar que la plaga se propagara más" (Macdonald $2017,2)$. Esto no sólo causó una gran impresión en las autoridades, también provocó la reorientación de los objetivos de atención de la salud, que ya no se enfocarían en el individuo sino en la comunidad. En ese contexto, la medicina occidental fue vista como un medio para alcanzar este objetivo (de la mano de las vacunas y el control ambiental), lo que socavó de manera decisiva la legitimación de la medicina nativa.

Según Lei (2000), los practicantes de ambas medicinas coexistieron durante décadas sin competir directamente entre sí, hasta que, al comienzo del periodo nacionalista — década de 1920-, los dos sistemas comenzaron una larga lucha que cambiaría el curso de la medicina tradicional. Mientras el Partido Nacionalista (Guómíndăng 国民党) intentaba llevar a cabo una agenda de modernización generalizada, fundó el Ministerio de Salud en Nanjing (primera vez en la historia del país que China tenía un centro administrativo nacional para hacerse cargo de los problemas relacionados con la atención médica). Durante la primera Conferencia Nacional de Salud Pública, celebrada en 1929, este ministerio, dirigido por médicos formados en Occidente, aprobó por unanimidad una propuesta para "abolir la medicina de estilo antiguo con el fin de eliminar los obstáculos a la medicina y la salud pública”" (Fang 2012, 6).

${ }^{9}$ Original: "Abolishing old style medicine in order to clear away the obstacles to medicine and public health". 
Resulta interesante ver cómo esta circunstancia adversa para la medicina tradicional es reconstruida como un hecho que fue resuelto con heroísmo y convicción. Así, como señala Farquhar (1994, 13): "Sorprendentemente, en una situación tan crítica, la comunidad de la medicina tradicional china aceptó el desafío y defendió su posición, y en lugar de desalentar a los médicos chinos tradicionales, la amenaza los movilizó en un movimiento masivo de medicina nacional que resultó un hito histórico para la disciplina".

Así, en el mismo año, los defensores de la práctica tradicional organizaron una asociación nacional (Instituto de Medicina Nacional) para combatir los desafíos legales e ideológicos. Esta confrontación "también constituyó un acto epistemológico que llevó a muchos médicos chinos a abrazar el discurso de la modernidad y a reformar la medicina china sobre la base de este discurso" (Lei 2000, 5), lo que condujo a la progresiva cientifización y modernización del sistema médico. El debate sobre la eliminación de la medicina tradicional motivó en la comunidad disciplinaria una autoevaluación que los "inspiró" a sistematizar la bibliografía, discutir teorías básicas e investigar sobre diferentes escuelas de pensamiento. Este movimiento político antagónico, y fuera de lo esperado, sentó las bases para una estandarización y un mayor desarrollo de este tipo de medicina (Liao 2017).

Como señalan algunos autores (Lei 2000; Liao 2017), aunque la llegada de la medicina occidental a China puede verse como un factor desalentador, finalmente promovió el desarrollo del sistema médico local. Como sostiene Liao $(2017,15)$ :

Bajo la presión de la medicina occidental, los médicos de la medicina tradicional china tomaron medidas para defenderla, promoverla y fortalecerla mediante la creación de escuelas, el inicio de programas de capacitación, la publicación de revistas, la organización de sociedades, la traducción de libros, el desarrollo de fármacos. Todos estos cambios muestran que la introducción de la medicina occidental en China, en cierta medida, promovió el desarrollo de la medicina tradicional. 
Con la fundación de la República Popular China comenzó un nuevo capítulo para la medicina tradicional. Después de años de guerra, el resultado fue un país devastado por décadas de pobreza, desnutrición y enfermedades endémicas. La nueva República "respondió a la difícil tarea que tenía por delante con la introducción de cambios sociales y económicos masivos [...] Se brindaron servicios básicos de atención a la salud y preventivos para todos como parte del sistema médico cooperativo" (Hesketh y Wei 1997, 1).

El presidente Mao Zedong apoyó el sistema de salud local con base en su valor cultural nacional y promovió el resurgimiento del interés del Estado en la medicina tradicional, al mismo tiempo que creía en el progreso realizado por la medicina occidental. Por otro lado, en uno de sus discursos (citado en Taylor 2011, 33) se ve reflejada una política pragmática de maximización y aprovechamiento de los recursos disponibles:

Los equipos de trabajo de salud de nuestra nación son grandes. Tienen que preocuparse por más de 500 millones de personas [incluidos los] jóvenes, viejos y enfermos [...] En la actualidad, los médicos de la medicina occidental son pocos y, por lo tanto, la gran mayoría de las personas, y en particular los campesinos, dependen de la medicina china para tratar enfermedades. Por ello, debemos luchar por la unificación completa de la medicina china.

En este contexto, en el Primer Congreso Nacional de Salud en 1950 se anunciaron cuatro pautas básicas para la organización de la atención médica: la medicina debe servir a los trabajadores, los campesinos y los soldados; la medicina preventiva debe tener prioridad sobre la medicina terapéutica; la medicina tradicional china debe integrarse con la medicina científica occidental, y el trabajo de salud debe combinarse con movimientos de masas (Farquhar 1994).

De este modo, se fomentó la posibilidad de lograr un acceso casi universal a la atención médica, lo que constituyó uno de los factores que explican la reducción en las tasas de 
mortalidad y morbilidad durante los años cincuenta y sesenta. Esta política fue acompañada de la mejora en las condiciones socioeconómicas y el énfasis en la prevención, por ejemplo, mediante campañas masivas de inmunización o acciones como la Campaña de las Cuatro Plagas (dirigida a eliminar moscas, mosquitos, ratas y gorriones) (Taylor 2011).

De acuerdo con la notable investigación dirigida por la antropóloga Judith Farquhar $(1994,11)$, el campo de la medicina tradicional surgió en su forma institucional moderna en 1949, sólo después de la fundación de la República Popular China. Más aún, las organizaciones contemporáneas de este tipo de medicina ubican sus inicios como entidades totalmente legítimas con la proclamación de Mao Zedong de 1955 de que "la medicina de nuestra patria es un gran tesoro". ${ }^{10}$

Entre mediados y finales de la década de 1950, se fundaron las primeras universidades de medicina tradicional, y las asociaciones profesionales anteriores a la Liberación ${ }^{11}$ se revitalizaron y ampliaron con apoyo público. La Oficina de Medicina China fue trasladada del Ministerio de Comercio al Ministerio de Salud, y de la misma manera los profesionales diversos y dispersos de la medicina tradicional, con sus pequeñas academias y clínicas familiares, se organizaron en una jerarquía nacional de instituciones clínicas y académicas en rápido crecimiento. La medicina tradicional adquirió una identidad profesional clara (Farquhar 1994).

De hecho, el Journal of Traditional Chinese Medicine ${ }^{12}$ se publicó por primera vez en $1955^{13}$ como la revista de medicina

${ }^{10}$ Original en inglés: treasure house.

${ }^{11}$ La fundación de la República Popular China en 1949 es un momento histórico conocido en el país como Liberación, y se habla de Preliberación en relación con los tiempos precedentes.

${ }^{12}$ Aún más, fue durante este periodo cuando surgió el concepto de "medicina tradicional china" para dar cohesión y unidad a esos grupos dispersos. Durante el periodo previo a la Liberación hubo un debate sobre el término más apropiado para referirse a ella: “medicina china” (zhōng y $y \bar{\imath}$ 中医), “medicina antigua” (jiùy $\bar{\imath}$ 旧医) o “medicina nacional” (guóyi 国医).

${ }^{13}$ En el contexto de la política de Reforma y Apertura, la primera edición en inglés se estrenó en 1981. 
tradicional más antigua, autorizada y de mayor circulación en China. En la misma línea, después de esta década, se establecieron instituciones de educación superior de esta clase de medicina en todo el país, lo que generó libros de texto de teoría unificada y un sistema estándar de materias, drogas y técnicas (Liao 2017). La medicina tradicional finalmente surgió como un sistema consolidado de la forma en la que más o menos se conoce hoy (Taylor 2011).

Más adelante en la línea temporal, durante la década de 1960, en el contexto de la Gran Revolución Cultural Proletaria (1966-1976), muchos médicos urbanos trabajaron como aldeanos y capacitaron a los locales, quienes se convertirían en los conocidos “médicos descalzos"14 (chìjiăo yishēeng 赤脚 医生). Como muchos autores comentan (Xu y Hu 2017; Wei 2013, entre otros), “aunque China había comenzado en 1958 a capacitar a médicos descalzos —en esencia, trabajadores mitad agricultores, mitad médicos,$-{ }^{15}$ no fueron institucionalizados ni entrenados masivamente, hasta que comenzó la Revolución cultural" (Wei 2013, 251). De hecho, el término “médicos descalzos" hizo su aparición oficial en 1968, en un artículo del Red Flag Journal. ${ }^{16}$ Los médicos descalzos tuvieron un papel clave en un sistema de salud más amplio, el Esquema Médico Cooperativo Rural, que operó entre 1968 y 1981 y se convirtió en el primer sistema nacional de seguro de salud primaria del país, que proporcionó atención a los campesinos, erradicó muchas enfermedades infecciosas y endémicas y mejoró sustancialmente la salud de las personas en las zonas rurales como nunca antes. Estos médicos también se convirtieron en factor fundamental de la integración de las medicinas china y occidental. Como plantean Xu y $\mathrm{Hu}(2017,140)$, durante la década de 1960 la política oficial de salud apuntó a la combinación de ambas, lo que tuvo como resultado una progresiva

${ }^{14}$ En inglés: barefoot doctors.

${ }^{15}$ Bànnóngbànyi 半农半医.

${ }^{16}$ Hóngqi 红旗. Fue una revista teórico-política publicada por el Partido Comunista Chino durante las décadas de 1960 y 1970. 
y gradual integración de estos saberes. Paralelamente, “el Estado también tomó medidas relevantes para apoyar el desarrollo de la medicina tradicional china. En su plan de estudios de capacitación, los médicos descalzos aprendieron tanto la medicina china como la occidental, así como la integración de las dos”.

Al final de este periodo, más de un millón de aldeanos (hombres y mujeres) ${ }^{17}$ recibieron capacitación, cultivaron hierbas medicinales y brindaron atención a la comunidad a muy bajo costo. Desde una perspectiva internacional, este movimiento sanitario fue considerado un ejemplo de excelencia en atención primaria de la salud y se convirtió en modelo para el mundo. Según la Organización Mundial de la Salud (2008), los médicos descalzos mejoraron radicalmente el acceso a la atención médica en las comunidades rurales de China y fueron una gran inspiración para el movimiento de atención primaria de salud que condujo a la conferencia en Alma-Ata, en la antigua República Soviética de Kazajstán en 1978: “estos trabajadores de la salud vivían en la comunidad a la que servían, centrados en la prevención en lugar de la cura mientras combinaban medicinas occidentales y tradicionales para educar a las personas y proporcionar un tratamiento básico”.

Más allá de estos avances, los grandes centros académicos que se habían forjado y consolidado durante el periodo anterior fueron diezmados durante la Revolución Cultural. Como explica Farquhar (1994), una de las formas visibles de las políticas "antiexpertos" de este periodo fue, por ejemplo, que ningún periódico profesional de medicina tradicional fuera publicado durante esta década, o que las escasas revistas médicas que aparecieron concentraran exclusivamente el trabajo de los médicos descalzos.

Con la llegada de Deng Xiaoping y su proceso de Reforma y Apertura, la medicina tradicional recibió un nuevo impulso, unido a la política de las cuatro modernizaciones: industria,

${ }^{17}$ Más de la mitad eran mujeres (Wei 2013). 
agricultura, ciencia y tecnología, y milicia. Este periodo dinámico, caracterizado por el cambio de política nacional, benefició a las instituciones de la medicina tradicional a la par de otras unidades académicas y científicas (Farquhar 1994). En este marco, en 1982 el papel formal de este tipo de medicina en el sistema de salud chino fue reconocido en la Constitución y en el ámbito nacional. La igualdad de estatus de los dos sistemas médicos se acentuó en 1985 y la política de integración continuó, aunque con un énfasis diferente. Se crearon tres categorías distintas de médicos que se mantienen hasta la actualidad: chinos tradicionales, occidentales e integrados (Griffiths, Chung y Jin 2010).

Aún más, la medicina tradicional no sólo se fue consolidando y restableció su estatus en el país, con la misma posición que la biomedicina, sino que, hacia fines del siglo xx, se fue extendiendo gradualmente a los países occidentales. Como establece Lei (2000, 3): "En Occidente, la medicina china forma parte de una gran familia mundial de medicinas alternativas y también ha sido aceptada en cierta medida en los servicios generales de atención médica".

En este marco, la interacción entre los saberes médicos chinos y occidentales comenzó a aumentar significativamente hasta nuestros días, a escala tanto nacional como internacional.

\section{Encuentros contemporáneos: usos y apropiaciones en la China actual}

La posibilidad de realizar una estancia de investigación en China en 2018 le brindó a la autora de este artículo la oportunidad de experimentar de primera mano la dinámica y los encuentros entre los sistemas de salud biomédico y chino. Durante la estancia se asistió a dos hospitales en la ciudad de Hangzhou, provincia de Zhejiang, así como a la Universidad de Medicina China de Zhejiang y su Museo de Historia de la Medicina China 
en la provincia. ${ }^{18}$ También se visitaron dos de las farmacias y clínicas tradicionales más prestigiosas de la ciudad: Húqìngyútáng 胡庆余堂 y Huíchūntáng 回春堂 (y su museo). ${ }^{19} \mathrm{~A}$ partir de las experiencias, las entrevistas y las conversaciones informales realizadas en esos lugares, se escriben las siguientes líneas.

En principio, más allá de los intentos de integración muchas veces exitosos entre ambos sistemas, es innegable que la medicina occidental ha ganado protagonismo en los itinerarios terapéuticos de los ciudadanos chinos, ${ }^{20}$ sobre todo en los de la gente más joven. Por otro lado, constituye el sistema médico que con preferencia cubren los seguros médicos. Con todo y eso, en la China actual son muy frecuentes en las prácticas cotidianas, predominantemente de los adultos y los adultos mayores, las técnicas relacionadas con el conocimiento tradicional de la atención médica. Las más habituales son el consumo de medicinas a base de hierbas, la práctica de tàijíquán 太极拳 o qìōng 气功, así como la ingesta de alimentos o bebidas medicinales. Del mismo modo, un gran número de personas se acerca a los establecimientos de medicina tradicional en busca de soluciones para tratar sus problemas de salud, ya sea acudiendo a consultas clínicas o comprando los remedios naturales que allí se dispensan. En este sentido, el caso de las clínicas-farmacia Húqingyútáng y Huíchūntáng es paradigmático y refleja el papel vital que Hangzhou ha desempeñado durante mucho tiempo en el desarrollo de la medicina tradicional. Ambos establecimientos fueron fundados durante la dinastía Qing (en 1874 y 1649 respectivamente) y cuentan con su propio taller de

${ }^{18}$ Este museo, además, muestra la historia de la carrera desde sus inicios en 1953.

${ }^{19}$ El Museo de Medicina Tradicional China de Húqingyútáng abrió sus puertas en 1991 y es uno de los de más famosos en esta especialidad. La exposición se divide en cinco partes que incluyen, además de una introducción histórica, una exhibición de talleres farmacéuticos y herramientas tradicionales, así como muestras de plantas, animales y minerales.

${ }^{20}$ También llamado "carrera del enfermo" o "carrera del paciente" (Menéndez 2003), este concepto alude a la trayectoria que despliegan los sujetos en la búsqueda de una respuesta a sus problemas de salud. 
fabricación de medicamentos, una tienda de medicinas y varios consultorios donde se ofrecen tratamientos herbolarios y de acupuntura (entre otras técnicas).

Como refirieron los informantes consultados durante la estancia en China, los itinerarios terapéuticos de la mayoría de los chinos, al menos en las zonas urbanas, oscilan entre la biomedicina y la medicina tradicional, ya sea porque acuden a este tipo de clínicas o porque realizan prácticas afines de autoatención en sus hogares y en su vida cotidiana. Resulta interesante mencionar que estos espacios, donde a simple vista pareciera fácil caer en una esencialización o romantización de "lo tradicional", se intersecan constantemente con los avances en materia tecnológica y virtual. Un ejemplo es la presencia en todos los consultorios de visores para placas radiográficas (lo que muestra una intersección con la biomedicina), o la modificación de técnicas tradicionales por la incorporación de aparatología (como en la moxibustión o la acupuntura). Del mismo modo, la comunicación por teléfonos móviles o celulares parece dominar la interacción entre pacientes y médicos. Así, códigos $\mathrm{QR}$ por doquier hacen crujir cualquier imagen maniquea de una China tradicional detenida en el tiempo (véase imagen 1). En el caso observado, los códigos QR permiten ingresar a la página web institucional (http://www.fhct.com/) y, de esta forma, se accede rápidamente a la información general de la institución y al currículo de los médicos, y es posible solicitar una cita en línea, entre otras opciones.

Además de la apropiación diversa de saberes y prácticas en la configuración de los itinerarios terapéuticos de muchos ciudadanos chinos, los encuentros entre sistemas médicos se observan también en los mismos establecimientos. En este sentido, "en la mayoría de los centros de salud y clínicas, la medicina tradicional china y la medicina occidental se practican juntas" (Griffiths, Chung y Jin 2010, 387). Esto va en contra de la creencia tan difundida en Occidente de que en China estos sistemas existen como entidades discretas, sin o con escaso contacto entre ellos. Por el contrario, como lo confirmó una 


\section{IMAGEN 1.}
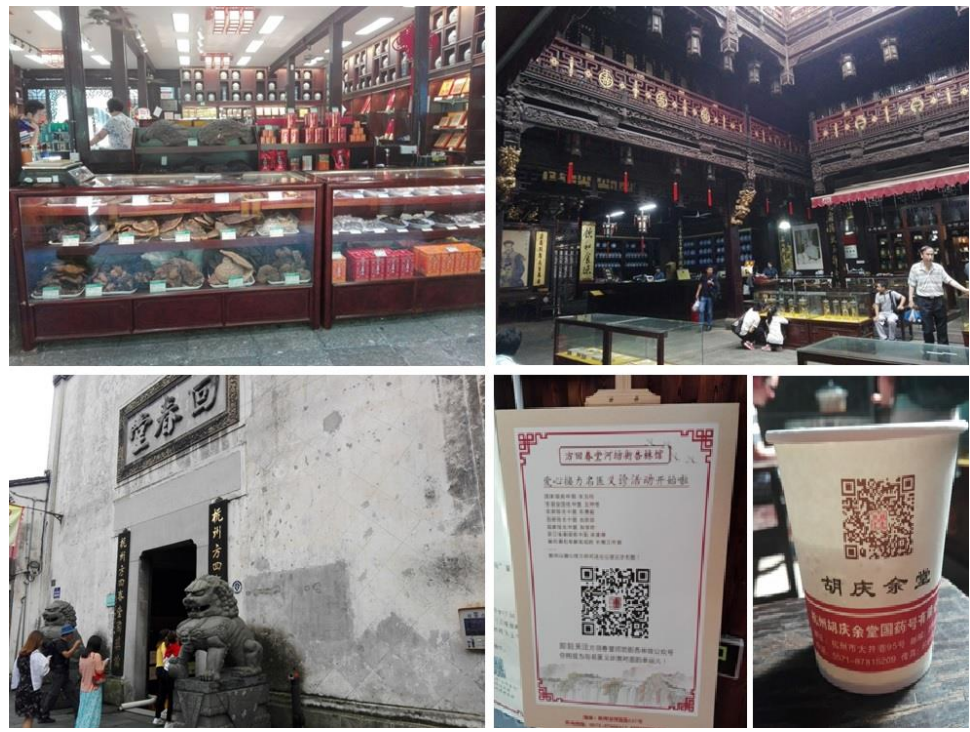

A la izquierda abajo, una de las entradas a la farmacia Huíchūntáng, fundada en 1649. Se puede ver a los lados de la entrada a los llamados "Leones de Fu" o "Leones chinos", poderosos animales míticos a los cuales se les atribuye la función de protección y seguridad. Arriba, salas destinadas a la exhibición y la venta de medicación natural, por peso o envasada. A la derecha abajo, ejemplos de la recurrencia de los códigos $Q R$ (a la izquierda en un banner, a la derecha en vasos donde se sirve té rojo dispensado gratuitamente). Fotografías de María Florencia Incaurgarat, 2018.

profesora de la Universidad de Medicina China de Zhejiang, ${ }^{21}$ todos los médicos están capacitados en ambos sistemas: los que estudiaron medicina occidental tuvieron un porcentaje de seminarios y práctica de medicina tradicional, y viceversa. ${ }^{22}$

${ }^{21}$ Creada en 1953, esta universidad multidisciplinaria especializada en medicina tradicional cuenta con más de 13000 estudiantes de tiempo completo, y ha sido pionera en el campo de los programas de medicina china al ofrecer programas de licenciatura, maestría, doctorado y posdoctorado desde entonces.

${ }^{22}$ Como afirma Macdonald (2017, 3): "desde la década de 1950, todos los estudiantes de medicina tienen que estudiar medicina moderna y tradicional. Aquellos 
De la misma manera, la derivación entre médicos es homóloga a la que sucede entre especialistas de la biomedicina (por ejemplo, entre un neurólogo y un traumatólogo), ya sea que decida el profesional, o bien que consulte al paciente sobre qué tipo de medicamento o tratamiento es preferible para el abordaje de alguna dolencia específica.

Esta relación dialógica entre saberes fue evidente en el Hospital Provincial de Medicina Tradicional China de Zhejiang. Fundado en 1931, es un hospital integral de grado 3 clase A, ${ }^{23}$ que ofrece tratamiento médico, educación médica, investigación científica y rehabilitación, con una marcada impronta médica tradicional. También es el primer hospital afiliado y el primer colegio clínico de la Universidad de Medicina China de Zhejiang. El hospital es conocido en el país por su larga historia, por contar con la mejor capacidad técnica de la provincia de Zhejiang, y por sus ventajas al combinar la medicina china con la occidental. Como narran en su sitio web (www.chinatcm. org/), después de una extensa práctica clínica, el hospital ha desarrollado tres pilares técnicos en los campos de la medicina tradicional, la medicina occidental y la combinación de ambas. Asimismo, el hospital tiene dos tipos de farmacias: occidental (xìyào pèifāng 西药配方) y china (zhōngyào pèifāng 中药配 方), dispuestas una al lado de la otra (véase imagen 2). Esta última es la farmacia de medicina tradicional más grande de la

estudiantes que deseen practicar la medicina tradicional deben estudiar los conocimientos modernos relevantes de anatomía, fisiología, etc., mientras que aquellos que desean practicar la medicina moderna tienen que asistir a conferencias sobre diversos aspectos tradicionales".

${ }^{23}$ En China, los hospitales públicos se clasifican de acuerdo con un sistema de tres niveles llamado Sānjíshídèng 三级十等 (tres grados, 10 niveles). Cada grado representa la capacidad del hospital para proporcionar atención y educación médica y realizar investigaciones; el nivel terciario representa el máximo desarrollo de estas habilidades. Además, el criterio de las letras A, B o C (jiăděng 甲等, y̌̃děng 乙等, b̌nngděng 丙等) subdivide los tres grados y configura diez niveles diferentes según el tamaño del establecimiento, la calidad médica, el equipamiento y la tecnología del hospital, entre otros. El décimo nivel está reservado para los hospitales más especializados (3AAA). 


\section{IMAGEN 2}

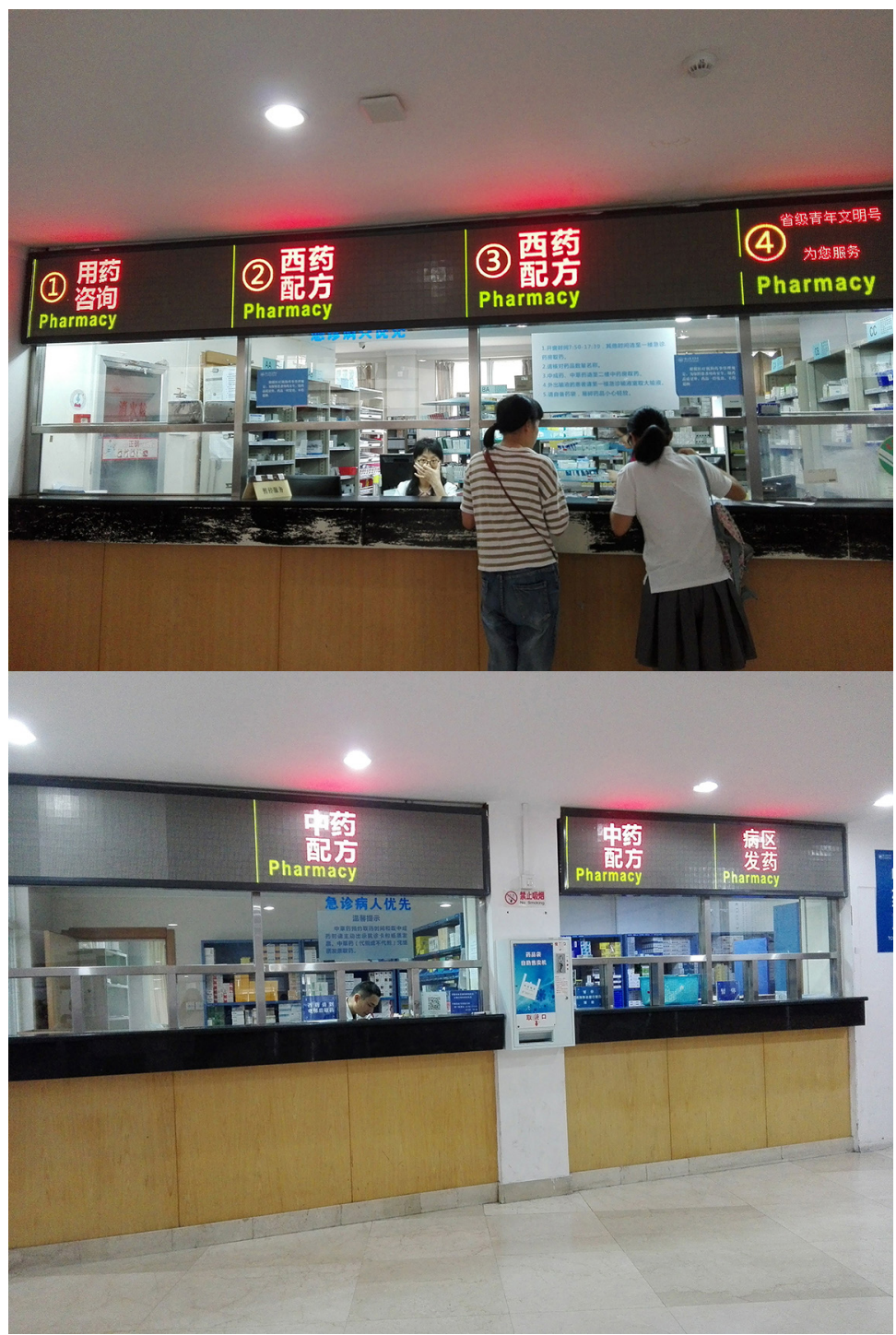

Arriba: farmacia de medicina tradicional china. Abajo: farmacia de medicina occidental. Fotografías de María Florencia Incaurgarat, 2018. 
provincia, con una producción diaria de más de 1000 fórmulas (alrededor de $4000 \mathrm{~kg}$ ).

En cuanto a los servicios ofrecidos en el hospital, se encuentran dispuestos como en los biomédicos, es decir, sectorizados por pisos o zonas individualizadas. En esos distintos sectores, además de las especialidades biomédicas (cardiología, neurología, psiquiatría, etc.), se cuenta con servicios específicos de la medicina tradicional, entre otros: acupuntura, departamento de tuina y medicina interna.

Con respecto a la elección de los servicios tradicionales o los biomédicos, los informantes consultados durante el programa, así como la bibliografía de referencia, revelaron las siguientes racionalidades. Por un lado, mientras que la medicina occidental es adecuada para encontrar soluciones rápidas, la tradicional es más lenta, pero más efectiva. De esta manera, existe un amplio consenso en que la medicina occidental no es apropiada para tratar ciertas enfermedades. Por el contrario, se cree que es más eficaz en el tratamiento de padecimientos en etapa aguda, como enfermedades coronarias, tuberculosis, hepatitis, cáncer, fracturas, problemas digestivos graves, entre otros. Se considera que la medicina tradicional es adecuada para la recuperación/ rehabilitación y como método de prevención y promoción de la salud, así como para el tratamiento de enfermedades crónicas. En este sentido, se cree que produce mejoras en el sistema inmunitario, efectos antivirales y antiinflamatorios, equilibrio entre cuerpo y mente, alivio del dolor y reducción del colesterol, entre otros (Green et al. 2006; Incaurgarat 2017, 188).

Además de esto, la elección de la medicina tradicional se basa en la creencia de que, aunque la medicina occidental es más rápida en el tratamiento de enfermedades agudas, genera diversos efectos adversos. Así, por lo general, en la búsqueda de una respuesta rápida, los servicios occidentales son los más demandados. Pero, a diferencia de lo que se podría suponer, las personas chinas no ven este desbalance como una amenaza para el futuro de la medicina tradicional. Afirman que, más allá 
de las razones mencionadas sobre los beneficios de ésta, también se solicita con frecuencia cuando la primera no encuentra una solución adecuada, o como complemento para contrarrestar los efectos no deseados de la biomedicina.

En cuanto a la combinación de los dos sistemas en un mismo tratamiento, hay un número creciente de estudios que trabaja en la aplicación fisiológica y biológica de ambos a la misma enfermedad (Schnell 1989; Griffiths, Chung y Jin 2010; Dong 2013; Li et al. 2016), con algunos resultados exitosos, relacionados por ejemplo con la reducción de los efectos secundarios de los remedios y las intervenciones occidentales. La integración de estos sistemas sigue siendo muy activa tanto en la investigación como en la práctica clínica y es considerada como la "nueva medicina del siglo xxi".

\section{La medicina tradicional china en el capitalismo global y la nueva era}

Como se ha visto, el sistema de salud de China ha sufrido numerosos y considerables cambios, en especial en las últimas décadas, con encuentros cada vez más variables y en escenarios cada vez más globales. Para finalizar este artículo, se ahondará, por un lado, en el papel de la medicina tradicional ante el crecimiento económico chino en lo que concierne a la planificación sanitaria gubernamental, $y$, por otro, en la medicina tradicional como una commodity y fuente de soft power en proyectos económicos a gran escala, como la iniciativa One Belt One Road (OBOR). Estas últimas reflexiones apuntan a cuestionar las perspectivas (sobre todo de sentido común) que ven la medicina tradicional como una entidad discreta, con límites definidos, y a desvelar que, por el contrario, se encuentra en constante modificación y renegociación no sólo de sus fronteras, sino también de su estatus nacional y mundial.

Como es bien sabido, el gran crecimiento de China en los últimos años ha mejorado el nivel de vida en la mayoría de 
los estratos sociales. Según la Agencia Sueca para el Análisis de la Política de Crecimiento, este factor,

en combinación con las fuertes necesidades de una atención médica mejor y más inclusiva, ha llevado a China a embarcarse en un importante programa de reforma de salud, con el objetivo de establecer un sistema universal de seguridad sanitaria. El nuevo sistema no es sólo un sistema de cuidado de la salud, también reconoce el impacto del medio ambiente, el estilo de vida y las circunstancias socioeconómicas en la salud (Growth Analysis 2013, 7).

El programa China Saludable en 2020 fue anunciado en 2009 por Chen Zhu, jefe del Ministerio de Salud, y consiste en un plan del gobierno para proporcionar atención médica básica e igualitaria a todos sus ciudadanos para 2020, en áreas rurales y urbanas, así como para promover estilos de vida saludables. En la misma sintonía, China Saludable en 2030, lanzado en 2016 después de la visita de Xi Jinping a la Conferencia Nacional de Salud y Bienestar de China en Beijing, amplía estos objetivos y abarca otros nuevos. En este caso, también es obligatorio construir entornos saludables, desarrollar una industria farmacéutica sólida basada en la innovación tecnológica médica y garantizar la seguridad de los alimentos y los medicamentos, entre otros (Tan, Liu y Shao 2017). Para promover estilos de vida saludables, la medicina tradicional es considerada por estos planes como un recurso valioso, sobre todo en lo concerniente a la prevención y el manejo de enfermedades crónicas. Como se establece en el esquema de China Saludable en 2030, para ese momento la medicina tradicional desempeñará un papel clave en la prevención y el tratamiento de enfermedades graves y en la rehabilitación. "Para esto será necesario, durante estos años siguientes, haber mejorado la capacidad de la medicina tradicional china, promovido la preservación y la innovación, e impulsado proyectos de servicios de mantenimiento preventivo y de salud basados en esta disciplina" (25). La integración y el apoyo de la medicina tradicional y la occidental también se incluyen en estos planes, al declarar que "los sistemas 
de prestación de atención médica deben integrarse, pasando de un modo de desarrollo extenso basado en la escala, a uno intensivo centrado en la calidad y la eficiencia" (13). En este sentido, se propone realizar esfuerzos en el desarrollo complementario de ambos sistemas médicos.

Fuera de las fronteras chinas, la medicina tradicional de ese país también ocupa un lugar relevante, aunque en mayor medida en materia comercial y de cooperación internacional. Por un lado, al ser considerada como parte de la "quintaesencia" nacional (junto con el daoísmo y el confucianismo), los medios de comunicación oficiales reconocen que ha gozado de gran popularidad en los países occidentales, y hoy en día es un servicio consumido principalmente por personas de clase media y como una alternativa a la medicina biomédica. En este sentido, desde las últimas décadas, su uso se ha extendido a más de 170 países, y el gobierno chino ha firmado aproximadamente 180 acuerdos de colaboración intergubernamental con otros países.

En el contexto de la iniciativa OBOR, la medicina tradicional es considerada un medio para colaborar con los países en desarrollo y sus sistemas de atención médica, así como una fuente de soft power. Esta intención se lee claramente en el periódico oficial China Daily, donde se establece que "a medida que la economía china se desarrolla continuamente a una velocidad relativamente alta y la fuerza nacional general de China crece constantemente, el soft power de la cultura china está atrayendo un mayor interés y una mayor atención", sobre todo en el extranjero (Liu y Mao 2015). Aunque se reconocen "obstáculos cognitivos y legales" por parte de las sociedades receptoras, se espera que la iniciativa OBOR sea una vía para sortearlos y lograr una mayor difusión de la medicina tradicional, ya que es en los países asociados a la franja económica donde se está "extendiendo" de manera más visible. En este sentido, para los países involucrados en la iniciativa, el gobierno chino ofrece "un marco de apoyo para la cooperación intergubernamental, que definitivamente ayudará a eliminar la barrera más grande, 
la barrera legal en la calificación de medicina tradicional china, la acreditación de un nivel educativo en ese ramo, el acceso a los fármacos y el seguro médico" (Song 2018).

Por otro lado, esta expansión global de la disciplina también está dando lugar a un crecimiento de la industria relacionada, ya que las grandes, medianas y pequeñas empresas chinas ahora también pueden participar en el comercio internacional. Según indica el China Daily: "Hay más de 2500 empresas especializadas en patentes de medicina china, con un valor de producción total de más de 600000 millones de yuanes, lo que representa aproximadamente un tercio de toda la industria médica. Hoy, las áreas de plantación de hierbas medicinales chinas en China ocupan más de 20 millones de mus". ${ }^{24}$

Por último, la medicina tradicional china también es vista desde una lógica economicista en términos de exportación, ya que se considera que "por poseer características simples, convenientes, fáciles y económicas, disfruta de un enorme potencial de mercado y de una amplia aceptación y apoyo del público en estos países [OBOR]” (Song 2018).

A partir de los aspectos desarrollados, se puede entrever la importancia que ha adquirido la medicina tradicional china en una escala cada vez más global, al ser parte de un proceso en constante movimiento y dejar abiertos interrogantes para su futuro desarrollo en la escena internacional.

\section{Reflexiones finales}

Como se observa en esta genealogía de encuentros entre saberes médicos, por más que la medicina tradicional china mantiene (o consolidó) su estatus tanto nacional como internacional, es innegable que, en definitiva, la que estableció los términos de la contienda fue precisamente la biomedicina, con sus criterios

${ }^{24}$ Medida de superficie equivalente a $800 \mathrm{~m}^{2}$. En este caso, 20 millones de mus equivalen a 1300000 hectáreas, aproximadamente. 
científicos occidentales. Como se describió -y como relata Zhan (2009) - , el conocimiento clínico y la autoridad de la medicina china se han reconfigurado a partir del encuentro con la biomedicina y sus parámetros científicos. Un claro ejemplo es el giro en la consagración de la investigación clínica en contraposición a los libros clásicos, y el cambio en lo referente a la formación de los médicos, antes orientada por "mentores" o "maestros", y ahora inscrita en universidades estatales. Es cierto, entonces, que estas dinámicas podrían ser entendidas dentro de "zonas de contacto" (Pratt, 1992, 33), es decir, en "espacios sociales donde culturas dispares se encuentran, chocan y luchan entre sí, a menudo en relaciones muy asimétricas de dominación y subordinación”. En otras palabras, estos encuentros se podrían entender en términos de “contacto", en tanto copresencia e interacción de relaciones de poder radicalmente asimétricas entre grupos antes separados histórica y geográficamente (no olvidemos el imperialismo británico y las guerras del Opio). Sin embargo, la perspectiva de Anna Tsing (2005) y su énfasis en la noción de "fricción" complementa esta mirada que corre el riesgo de caer en una comprensión universalista de las relaciones de poder entre dominados y dominadores, oprimidos y opresores. Así, la autora nos recuerda que la globalización es más imprevisible y menos "homogeneizadora" de lo que el sentido común muchas veces postula.

Con base en este postulado, se puede analizar el encuentro entre estos sistemas médicos no desde una lógica vertical, en la que la medicina occidental se haya impuesto sobre la tradicional, y ésta la haya recibido de modo pasivo. Por el contrario, como se describió en el artículo, el encuentro entre estos corpus de saberes produjo una "nueva geografía" (Faier y Rofel 2014) que no sólo modificó las formas de atender la salud y la enfermedad dentro de las fronteras territoriales, sino que se continúa proyectando a niveles cada vez más globales. 


\section{Referencias}

Dong, Jingcheng. 2013. "The Relationship between Traditional Chinese Medicine and Modern Medicine". Evidence-Based Complementary and Alternative Medicine 2013. https://doi. org/10.1155/2013/153148

FAIER, Lieba y Lisa Rofel. 2014. "Ethnographies of Encounter". Annual Review of Anthropology 43 (1): 363-377. https://doi. org/10.1146/annurev-anthro-102313-030210

FANG, Xiaoping. 2012. Barefoot Doctors and Western Medicine in China. Woodbridge: Boydell \& Brewer.

FARQUHAR, Judith. 1994. Knowing Practice: The Clinical Encounter of Chinese Medicine. Oxford: Westview Press.

Fu, Louis. 2014. "Hippocratic Medicine in China: Comparison with a $9^{\text {th }}$ Century Chinese Manual on Bone Setting”. Journal of Orthopaedics, Trauma and Rebabilitation 18 (2): 128-135. https:// doi.org/10.1016/j.jotr.2014.09.001

Green, Gill, Hannah Bradby, Anita Chan y Maggie Lee. 2006. “We are not Completely Westernised: Dual Medical Systems and Pathways to Health Care among Chinese Migrant Women in England”. Social Science E Medicine 62 (6): 1498-1509. https:// doi.org/10.1016/j.socscimed.2005.08.014

Griffiths, Sian, Vincent Chung y Jin Tang. 2010. "Integrating Traditional Chinese Medicine: Experiences from China". Australasian Medical Journal 3 (7): 385-396. https://doi.org/10.4066/ AMJ.2010.411

Growth ANALYsis. 2013. "China's Healthcare System: Overview and Quality Improvements” (Direct response 2013:03). Östersund: Swedish Agency for Growth Policy Analysis. http://www. tillvaxtanalys.se/in-english/publications/direct-response/directresponse/2013-05-20-chinas-healthcare-system-----overview-andquality-improvements.html

Hesketh, Therese y Xing Zhu Wei. 1997. "Health in China: Traditional Chinese Medicine: One Country, Two Systems”. British Medical Journal 315 (7100): 115-117 https://doi.org/10.1136/ bmj.315.7100.115

INCAURGARAT, María Florencia. 2017. “El ‘viento’ como agente generador de padecimiento. Reflexiones sobre el periodo de posparto 
en relación al 'pensamiento chino"'. Avá 29: 175-197. www.ava. unam.edu.ar/images/29/n29a07.pdf

JACKSON, Mark, ed. 2011. The Oxford Handbook of the History of Medicine. Oxford: Oxford University Press. https://doi.org/10.1093/ oxfordhb/9780199546497.001.0001

LEI, Sean Hsiang-lin. 2000. "When Chinese Medicine Encountered the State, 1928-1937". Tesis de doctorado. Universidad de Chicago. http://www.ihp.sinica.edu.tw/ medicine/active/years/ hl.PDF

LI, Deli, Shanshan Qiao, Duozhi Shi, Shiqi Zheng, Tengyu Wang y Rufeng Wang. 2016. "The Combination of Traditional Chinese Medicine with Western Medicine". Medicinal E Aromatic Plant 5 (6). https://doi.org/10.4172/2167-0412.1000e179

Liao, Yuqun. 2017. Traditional Chinese Medicine: Understanding its Principles and Practices. Beijing: China Intercontinental Press.

Liu Diangang y Mao Herong. 2015. “'One Belt and One Road' Strategy: Opportunities for International Communication of Traditional Chinese Medical Culture”. China Daily, 28 de enero de 2015. http://www.chinadaily.com.cn/culture/2015-01/28/ content_19430421.htm

Macdonald, Alexander. 2017. "Relationship of Modern and Traditional Schools of Chinese Medicine: Need it Remain Hostile?". International Journal of Complementary $\mathcal{E}$ Alternative Medicine 7 (2): 00220. https://doi.org/10.15406/ijcam.2017.07.00220

MENÉNDEZ, Eduardo. 2003. "Modelos de atención de los padecimientos: de exclusiones teóricas y articulaciones prácticas". Ciência ESaúde Coletiva 8 (1): 185-207. https://doi.org/10.1590/S141381232003000100014

Moreno García, Julia. 2018. China contemporánea: 1916-2017. $2^{\text {a }}$ ed. Madrid: Istmo.

Organización Mundial de la Salud. 2008. “China’s Village Doctors Take Great Strides”. Bulletin of the World Health Organization 86 (12): 909-988. https://doi.org/10.2471/BLT.08.021208

PRATT, Mary Louise. 1992. Imperial Eyes. Travel Writing and Transculturation. Londres: Routledge.

Puerta, José Luis. 2009. "El canon de medicina interna del Emperador Amarillo". Dendra Médica. Revista de Humanidades 8 (1): 100-106. https://dialnet.unirioja.es/servlet/articulo?codigo $=4369903$ 
SCHNell, James A. 1989. “The Merging of Traditional Chinese Medicine and Western Medicine in China: Old Ideas Cross-Culturally Communicated Through New Perspectives". Explorations in Ethnic Studies 12 (1): 41-45. https://doi.org/10.1525/ees.1989.12.1.41

Song, Jingli. 2018. "Boosted by Belt and Road Initiative, Spread of TCM Speeds up". China Daily, 4 de junio de 2018. http:// europe.chinadaily.com.cn/a/201806/04/WS5b14ab0fa31001b82571dfaf.html

TAN, Xiaodong, Xiangxiang Liu y Haiyan Shao. 2017. "Healthy China 2030: A Vision for Health Care". Value in Health Regional Issues 12: 112-114. https://doi.org/10.1016/j.vhri.2017.04.001

TAYlor, Kim. 2011. Chinese Medicine in Early Communist China, 1945-63: A Medicine of Revolution. Londres: RoutledgeCurzon. Tsing, Anna Lowenhaupt. 2005. Friction: An Ethnography of Global Connection. Princeton, NJ: Princeton University Press. https:// doi.org/10.1515/9781400830596

UnsChUld, Paul U. 2003. Huang Di nei jing su wen: Nature, Knowledge, Imagery in an Ancient Chinese Medical Text. Berkeley: University of California Press. https://doi.org/10.1525/9780520928497

WeI, Chunjuan Nancy. 2013. "Barefoot Doctors: The Legacy of Chairman Mao's Health Care”. En Mr. Science and Chairman Mao's Cultural Revolution, editado por Chunjuan Nancy Wei y Darryl E. Brock, 251-280. Lanham: Lexington Books.

Xu, Sanchun y Danian Hu. 2017. "Barefoot Doctors and the 'Health Care Revolution' in -Rural China: A Study Centered on Shandong Province”. Endeavour 41 (3): 136-145. https://doi. org/10.1016/j.endeavour.2017.06.004

Zhan, Mei. 2009. Other-wordly. Making Chinese Medicine through Trasnational Frames. Durham: Duke University Press. https:// doi.org/10.2307/j.ctv1198xrp

María Florencia Incaurgarat es doctora en antropología social (Instituto de Altos Estudios Sociales/Universidad Nacional de San Martín), docente universitaria (Universidad Nacional de Mar del Plata) y becaria doctoral del Consejo Nacional de Investigaciones Científicas y Técnicas (Conicet). Asimismo, fue becaria del Programa de Jóvenes Sinólogos Visitantes de la 
República Popular China (2018) y del Programa junTos, JICA, Japón (2017). Sus temas de estudio son antropología de la salud, migración china e interculturalidad y estudios sinológicos (cultura y salud).

https://orcid.org/0000-0002-0852-0735

flor.incaurgarat@gmail.com 